\title{
Amino Acid-based Poly(Ester Urea)s as a Matrix for Extended Release of Entecavir
}

\author{
Alexandra K. Abel ${ }^{a}$, Nathan Z. Dreger ${ }^{\mathrm{a}}$, Karissa Nettleton ${ }^{\mathrm{a}}$, Tiffany P. Gustafson ${ }^{\mathrm{b}}$, Seth \\ Forster $^{\mathrm{b}}$, Matthew L. Becker ${ }^{\mathrm{c}, \mathrm{d}, \mathrm{e}}$ \\ ${ }^{a}$ Department of Polymer Science, The University of Akron, Akron, Ohio 44325, USA
}

${ }^{b}$ Merck \& Co., Inc., Department of Pharmaceutical Sciences, 90 E. Scott Ave, Rahway, NJ, 07065, USA

'Department of Chemistry, Duke University, Durham, NC, 27708, USA

${ }^{\mathrm{d}}$ Department of Mechanical Engineering and Material Science, Duke University, Durham, NC, 27708, USA

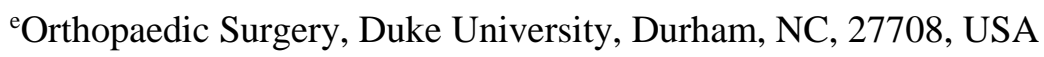

\section{E-mail: matthew.1.becker@duke.edu}

Declarations of Interest: ML Becker has filed provisional patent and PCT applications with the University of Akron Research Foundation. Some of these patents have been licensed to a startup company 21MedTech, LLC in which Dr. Becker and the University of Akron Research Foundation are equity holders.

\section{Table of Contents:}

${ }^{1} \mathrm{H}$ NMR of Monomers and Polymers

Size Exclusion Chromatography Eluograms

Thermal Analysis Data

UPLC Chromatograms

Release Profiles (total amount released)

Images of Extruded Filaments

Statistical Analysis of Cumulative Release

SEM Micrographs

X-Ray Microcomputed Tomography Cross Sections

Statistical Analysis of Porosity

Force vs. Displacement Plots

${ }^{1} \mathrm{H}$ NMR of Entecavir and Effluent Residues

Page
$2-5$
6
7
8
9
9
$10-12$
13
$14-15$
$16-18$
19
20




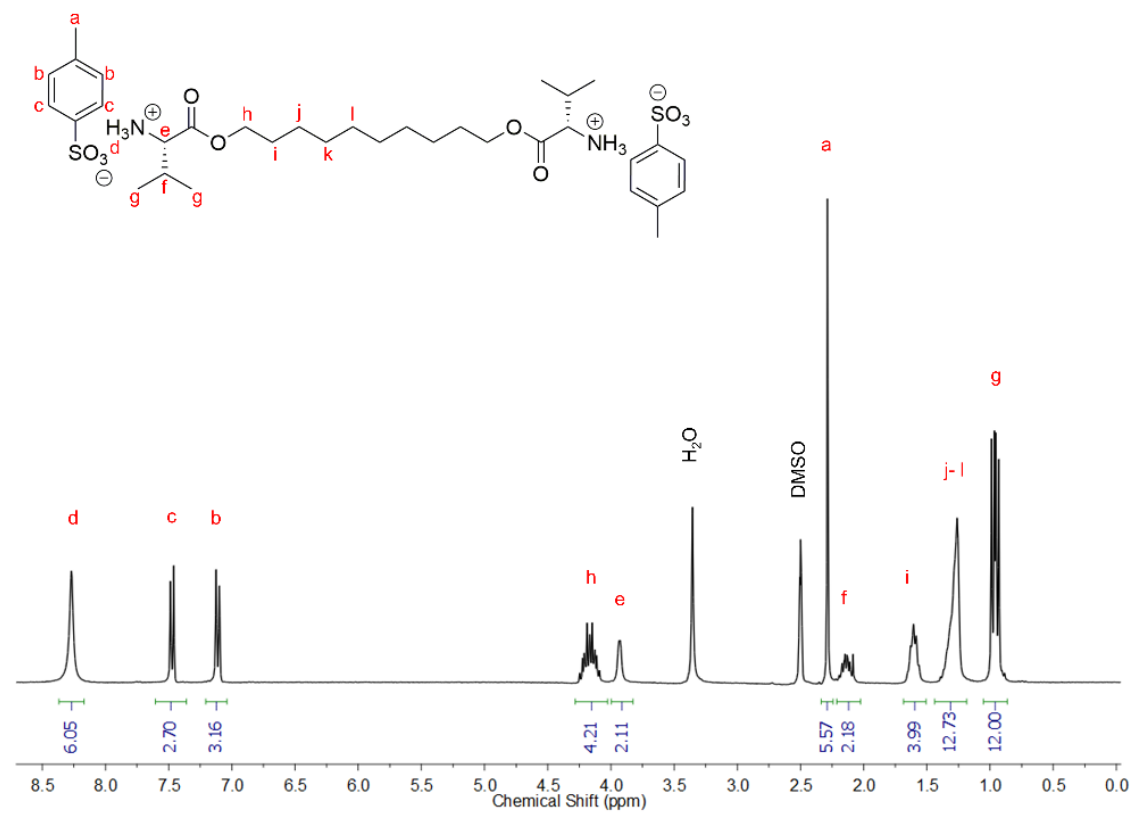

SI Figure 1. ${ }^{1} \mathrm{H}$ NMR spectra of 1-VAL-10 monomer confirms successful synthesis and purity due to the peak assignments.

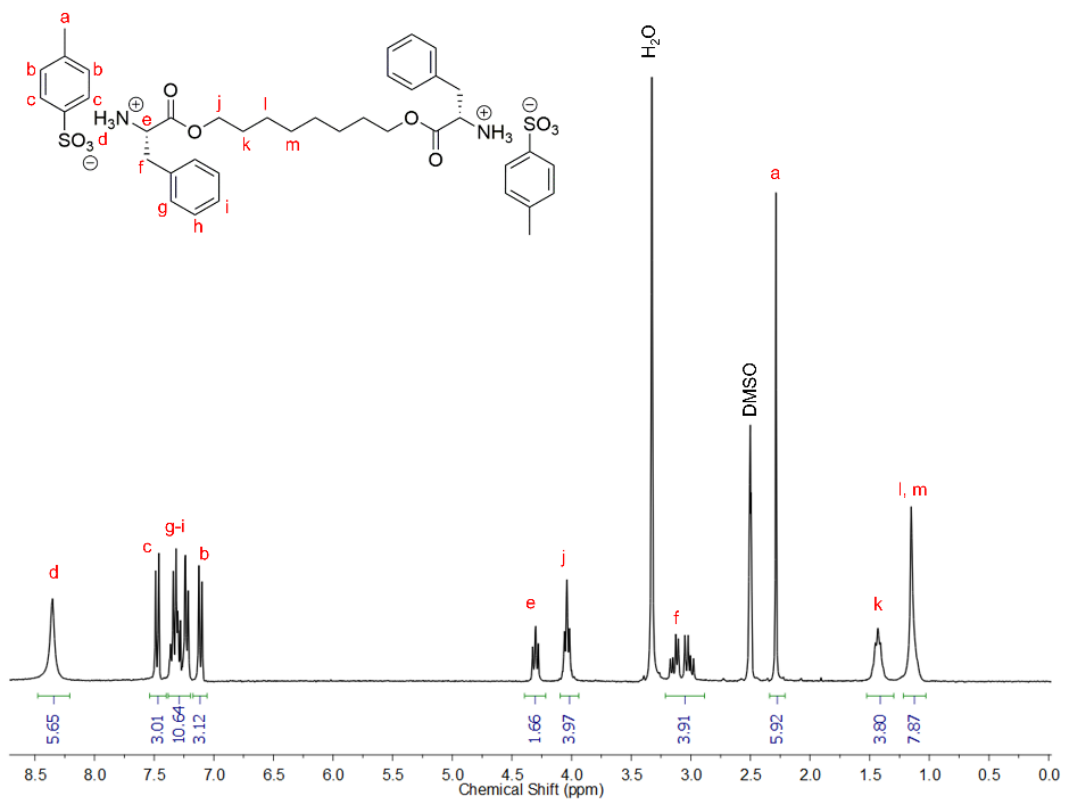

SI Figure 2. ${ }^{1} \mathrm{H}$ NMR spectra of 1-PHE-8 monomer confirms successful synthesis and purity due to the peak assignments. 


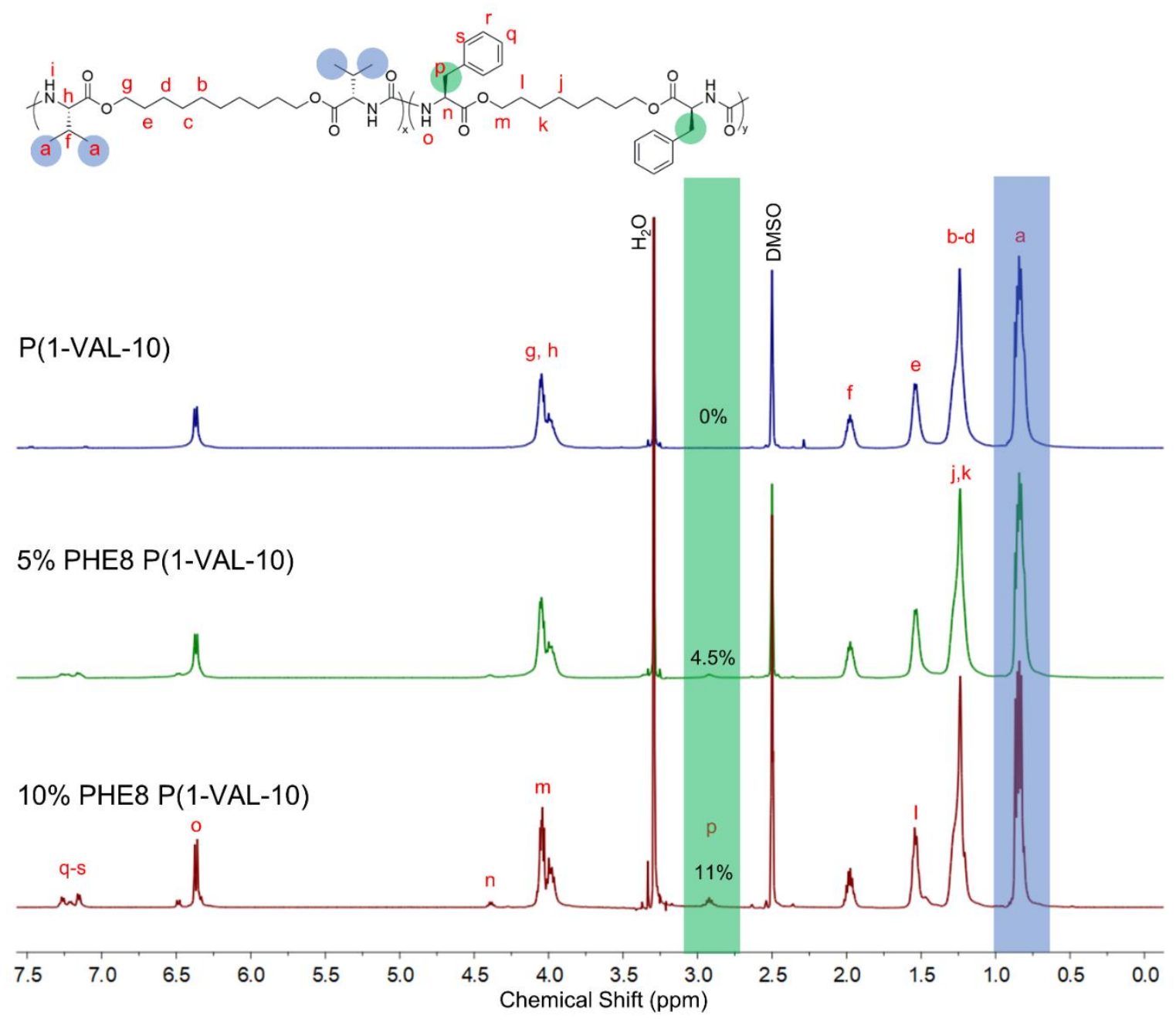

SI Figure 3. ${ }^{1} \mathrm{H}$ NMR spectra of $\mathrm{P}(1-\mathrm{VAL}-10)$, 5\% PHE8 $\mathrm{P}(1-\mathrm{VAL}-10)$, and $10 \%$ PHE8 P(1VAL-10) confirms successful synthesis and purity due to the peak assignments based on integration values. The ratio of PHE8 in each of the polymers is calculated by the corresponding resonances from peak "a" from the $L$-valine and peak "p" from the $L$-phenylalanine. As the molar ratio of the PHE8 in the polymer synthesis is increased, a higher ratio of PHE8 in the copolymers is observed. 


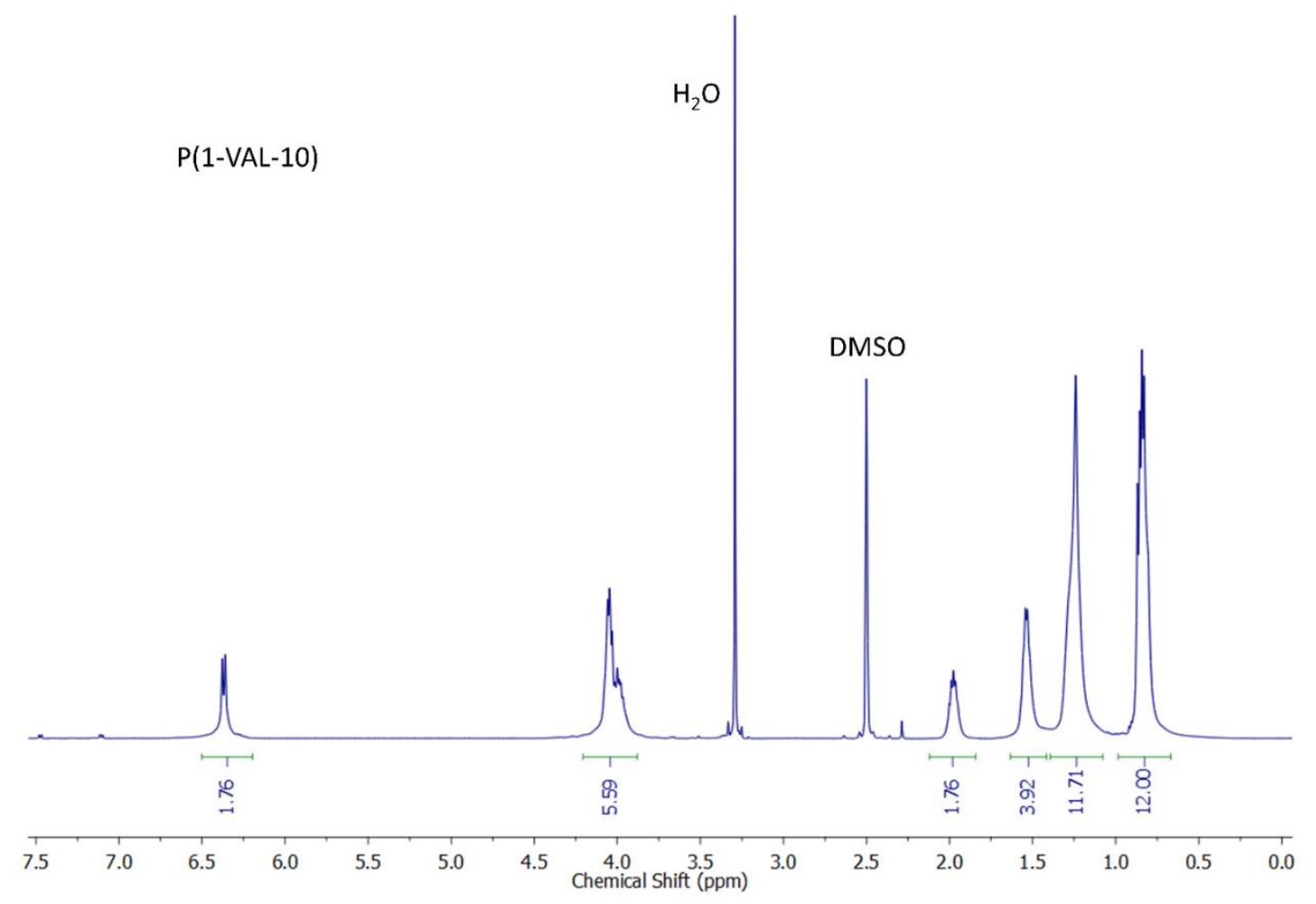

SI Figure 4. ${ }^{1} \mathrm{H}$ NMR spectra of $\mathrm{P}(1-\mathrm{VAL}-10)$ with corresponding integration values.

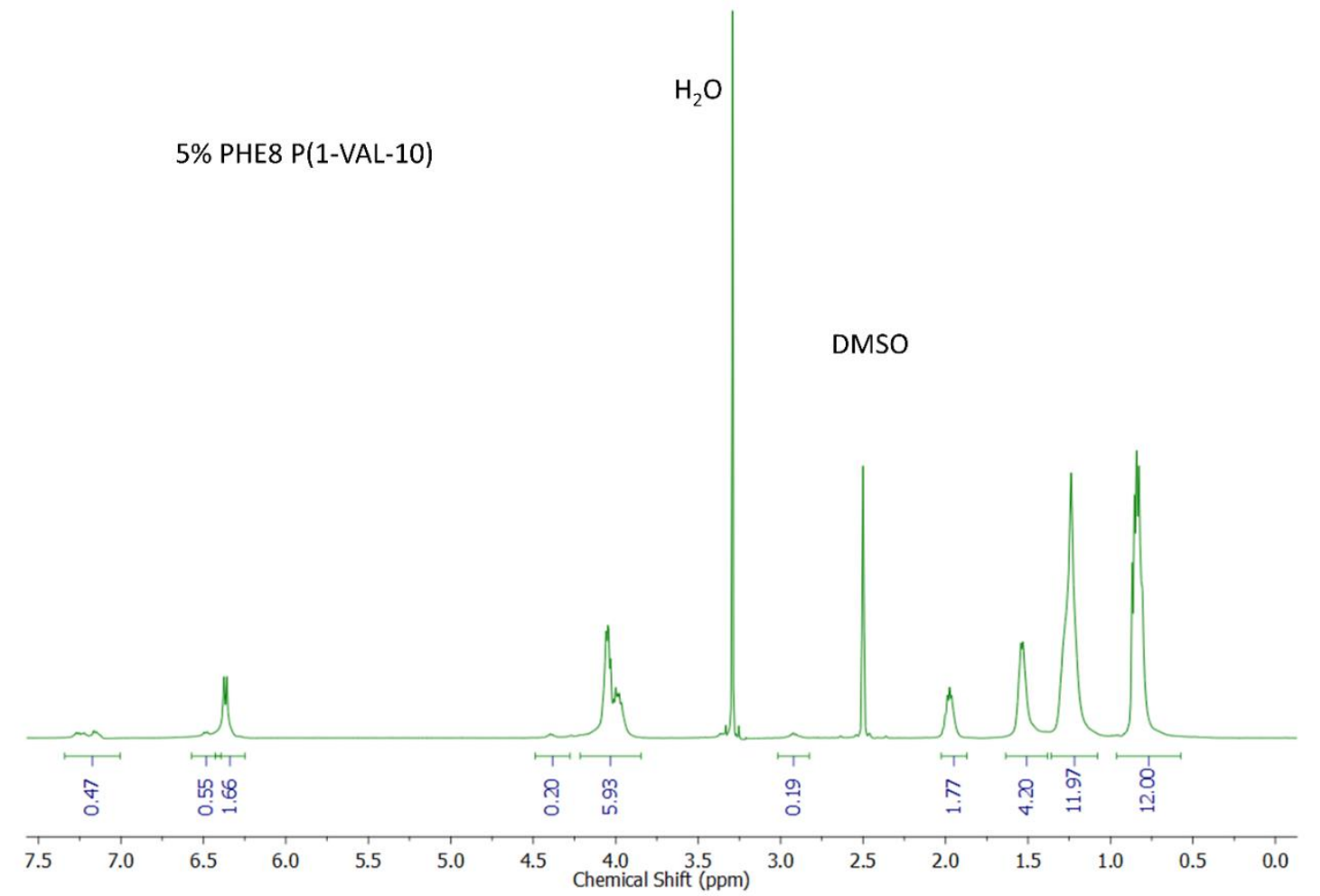

SI Figure 5. ${ }^{1} \mathrm{H}$ NMR spectra of 5\% PHE8 P(1-VAL-10) with corresponding integration values. 


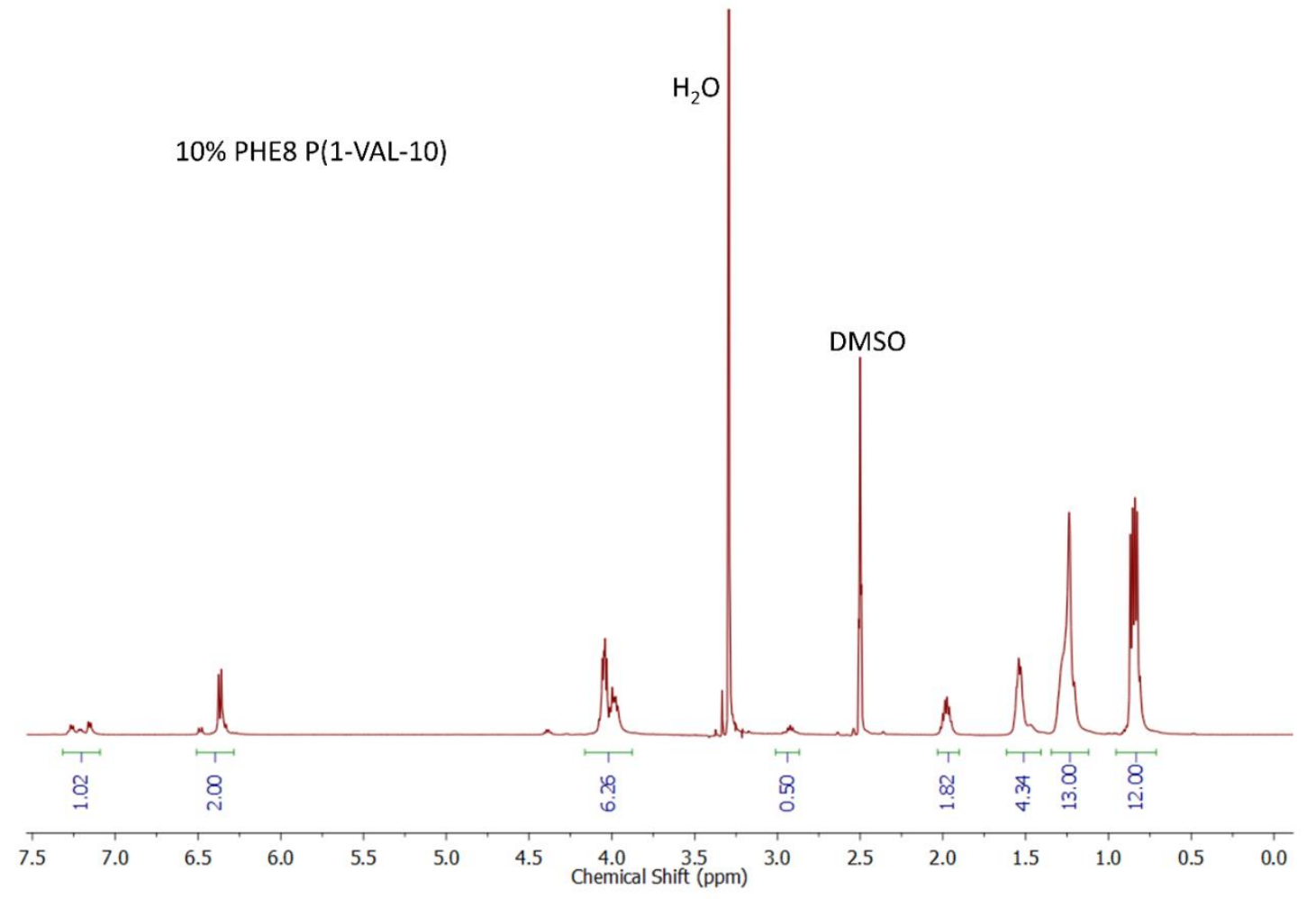

SI Figure 6. ${ }^{1} \mathrm{H}$ NMR spectra of $10 \%$ PHE8 P(1-VAL-10) with corresponding integration values. 
A

$P(1-V A L-10)$

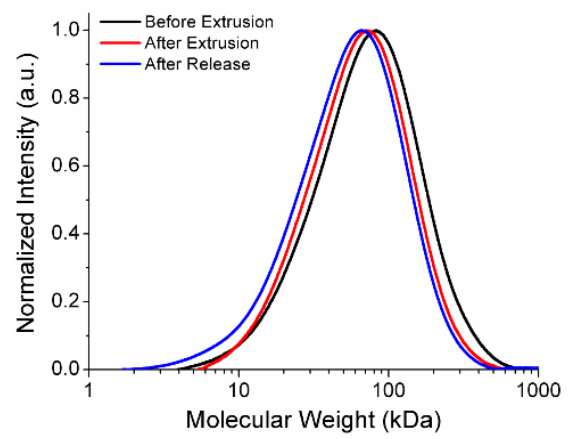

B 5\% PHE8 P(1-VAL-10)

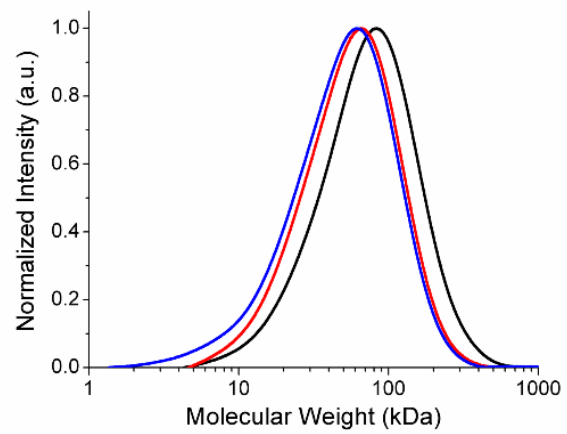

C $10 \%$ PHE P(1-VAL-10)

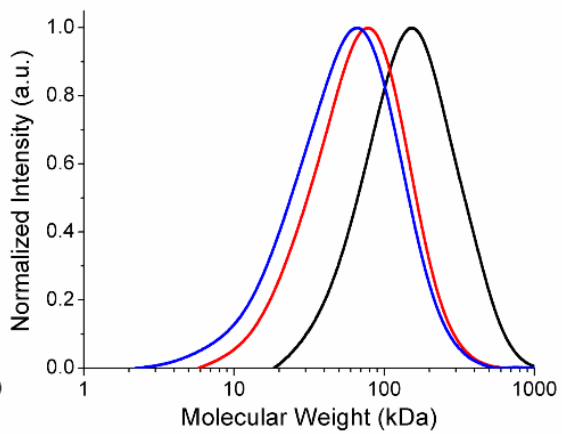

SI Figure 7. Molecular mass distributions of the pure polymer filaments $(A-C)$ before extrusion, after extrusion, and after the 4-week release period are shown. Molecular mass decreases after extrusion due to thermal degradation of polymer chains. A further decrease in molecular mass is observed after release due to hydrolytic cleavage of ester linkages within the PEU.
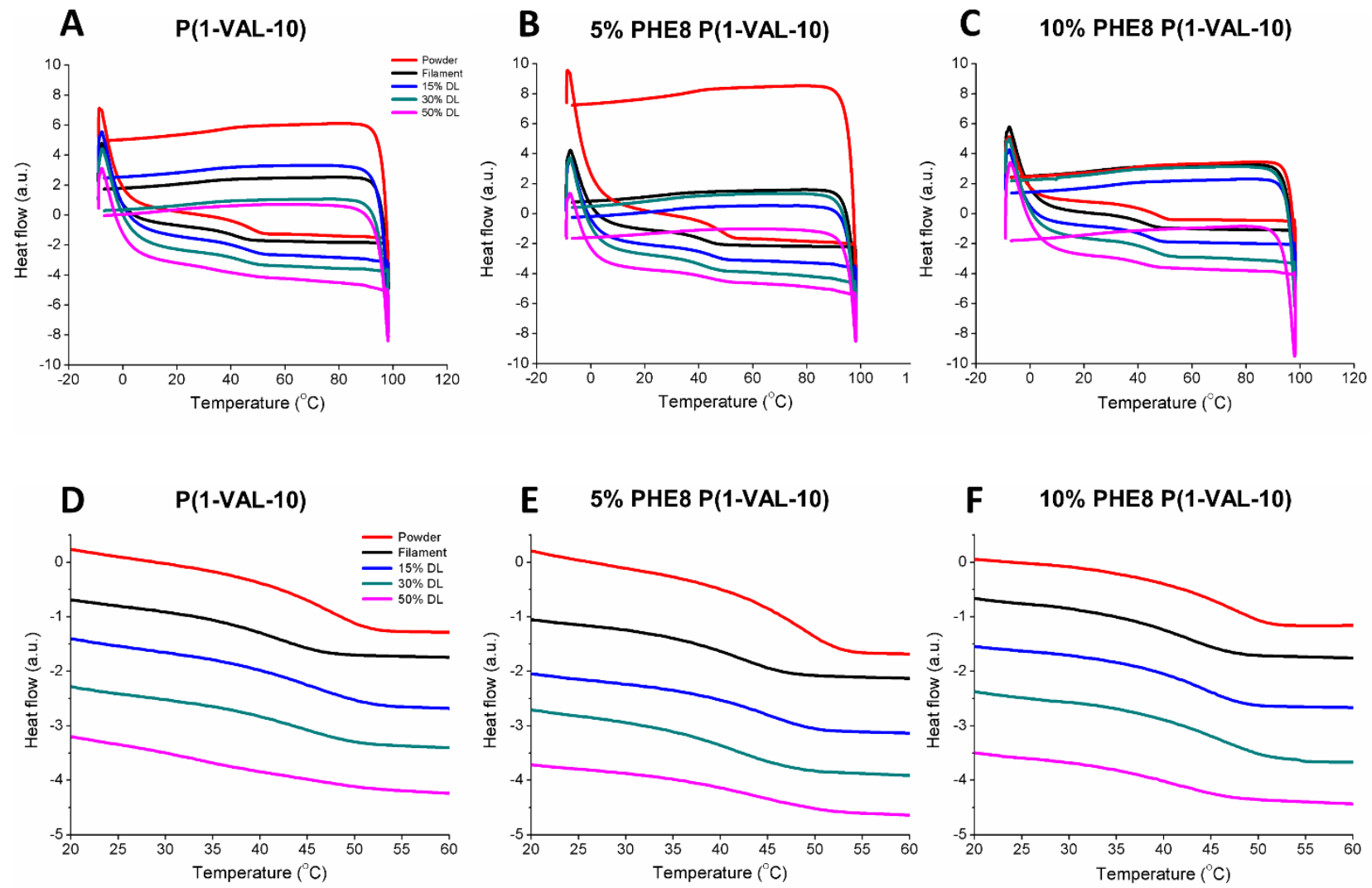

SI Figure 8. Differential scanning calorimetry was performed on the polymer powder before extrusion and on each of the subsequent extruded filaments. The second full heating and cooling cycle is shown in $\mathrm{A}-\mathrm{C}$. Enlarged $\mathrm{T}_{\mathrm{g}}$ regions are shown in $\mathrm{D}-\mathrm{F}$. 


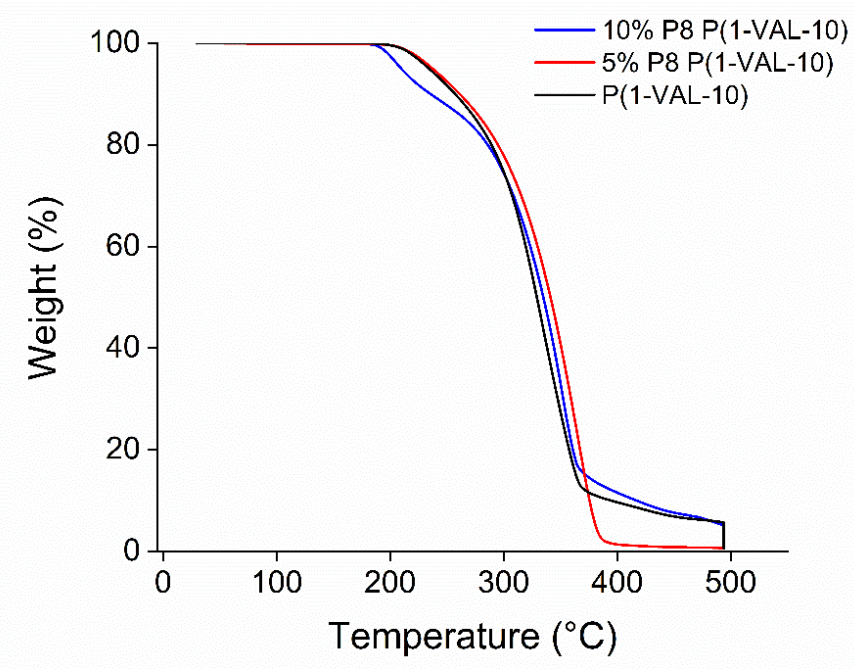

Figure 9. Thermal gravimetric analysis was used to analyze the degradation temperature $\left(\mathrm{T}_{\mathrm{d}}\right)$ of P(1-VAL-10), 5\% PHE8 P(1-VAL-10), and 10\% PHE8 P(1-VAL-10)
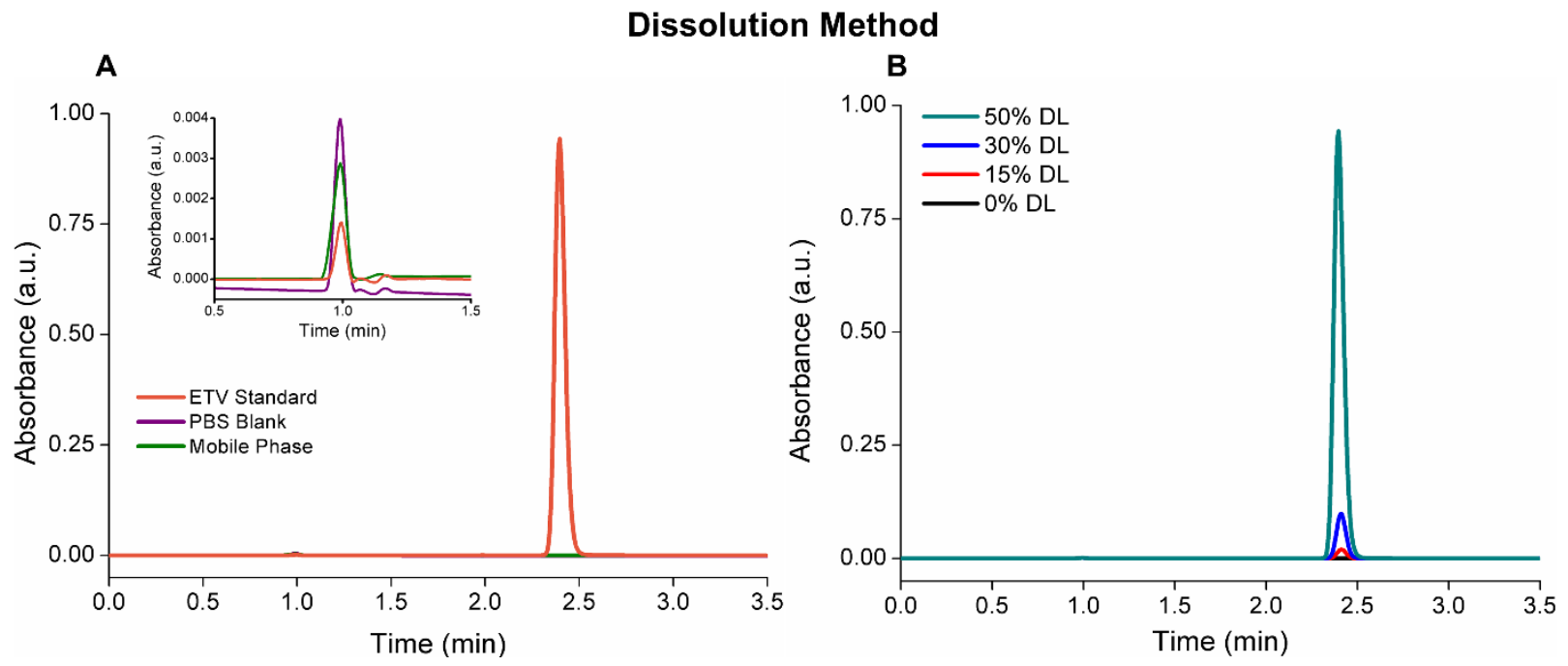

SI Figure 10. Representative UPLC chromatograms of the dissolution method. Each injection shows a monomodal peak at $\sim 2.4$ minutes which confirms the presence of entecavir. There was no drug degradation observed over time. A) The blank and standard injections show the column void volume peaks around 1 minute and B) demonstrates the effect of increased drug content on absorbance. 


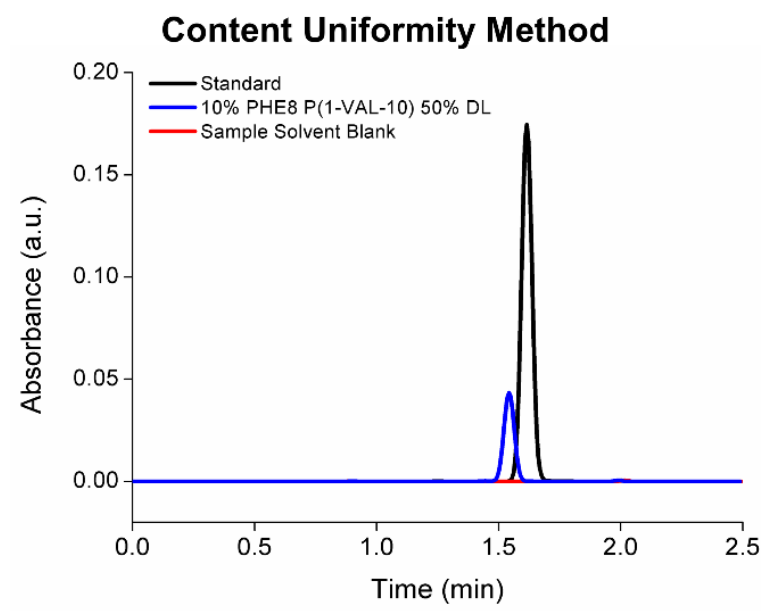

SI Figure 11. Representative UPLC chromatograms of the content uniformity method. The slight shift in sample retention time when compared to the standard can be attributed to the presence of polymer in the injection.
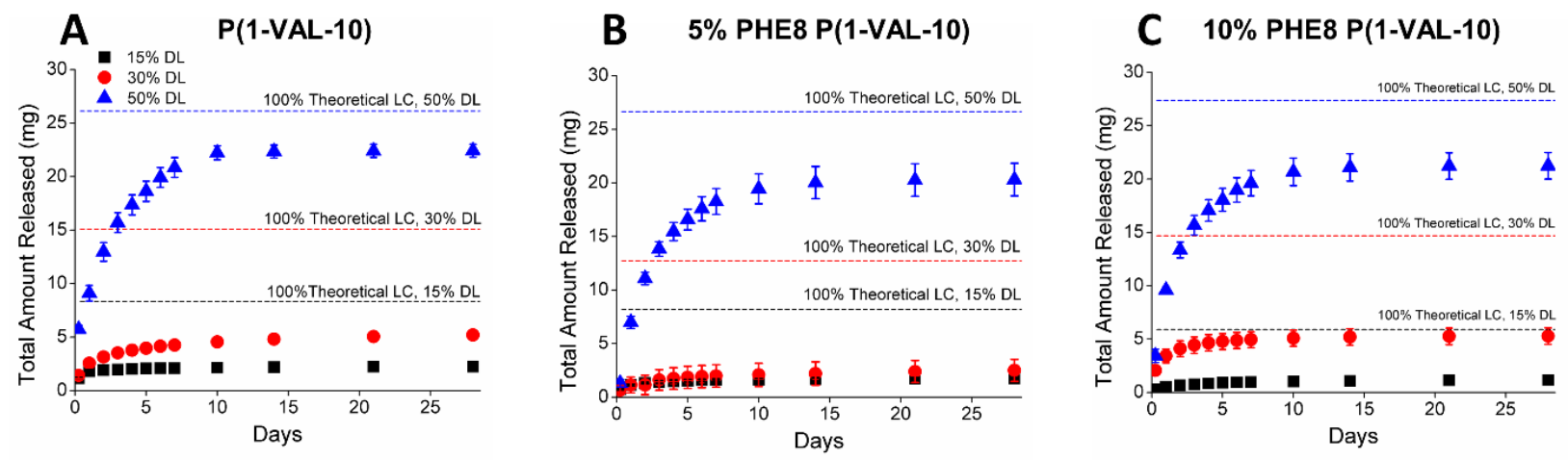

SI Figure 12. The release profiles ( $n=5)$ at different targeted drug loadings of $A) P(1-V A L-10)$, B) 5\% PHE8 P(1-VAL-10), and C) 10\% PHE8 P(1-VAL-10). The rate of release increases with increasing $\%$ drug loading. The 50\% DL filaments each release $100 \%$ of their DL after to 4 -week period when using the actual label claim; whereas, the 15\% DL filaments released below $50 \%$ and the $30 \%$ DL filaments released below $80 \%$. 


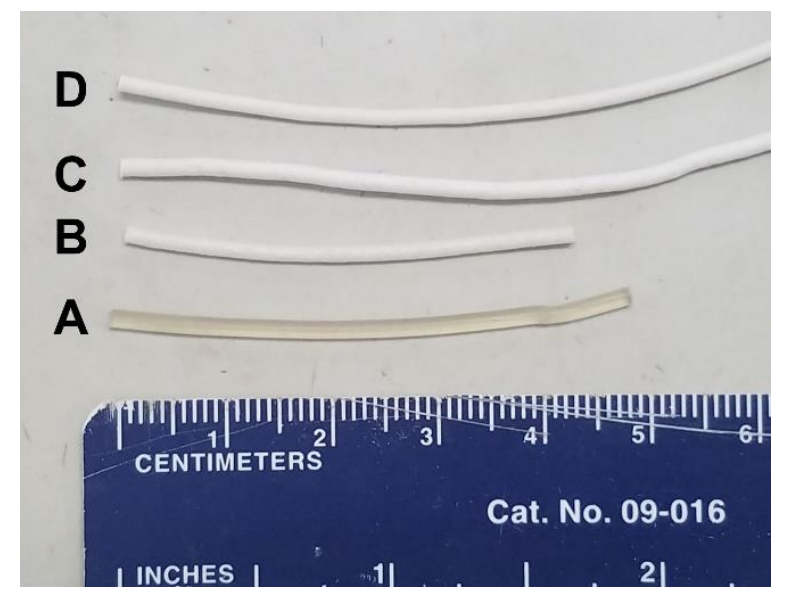

SI Figure 13. 10\% PHE8 P(1-VAL-10) filaments extruded with A) 0\% DL, B) 15\% DL, C) $30 \%$ DL, and D) $50 \%$ DL.

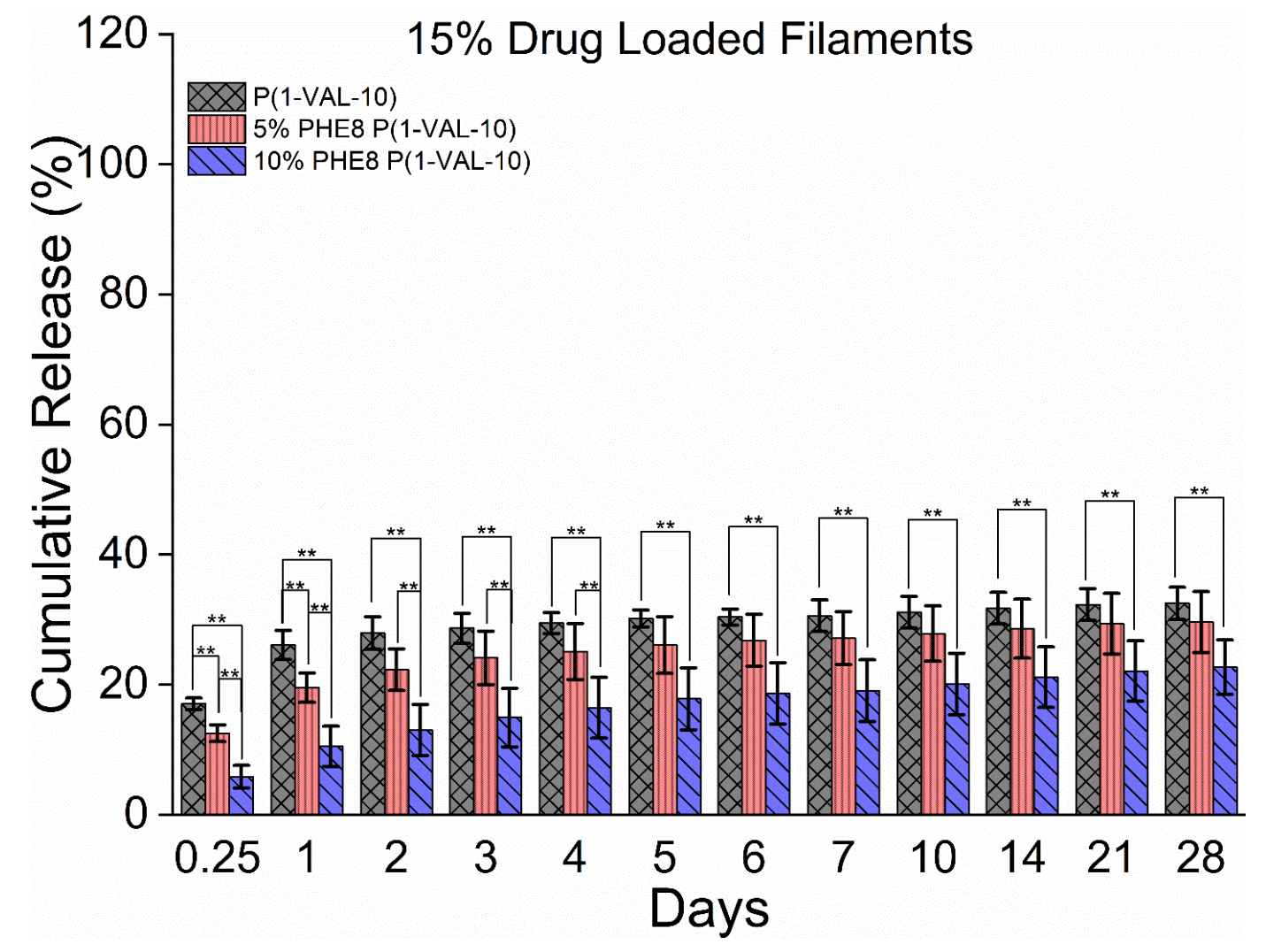

SI Figure 14. Statistical analysis ( $* * \leq 0.01$ significance value) of release values $(n=5)$ of the $15 \%$ DL filaments at each timepoint using a two-sample t-test assuming equal variance shows a significant difference in cumulative release over the 4 week trial period between the samples made with P(1-VAL-10) and 10\% PHE8 P(1-VAL-10). 


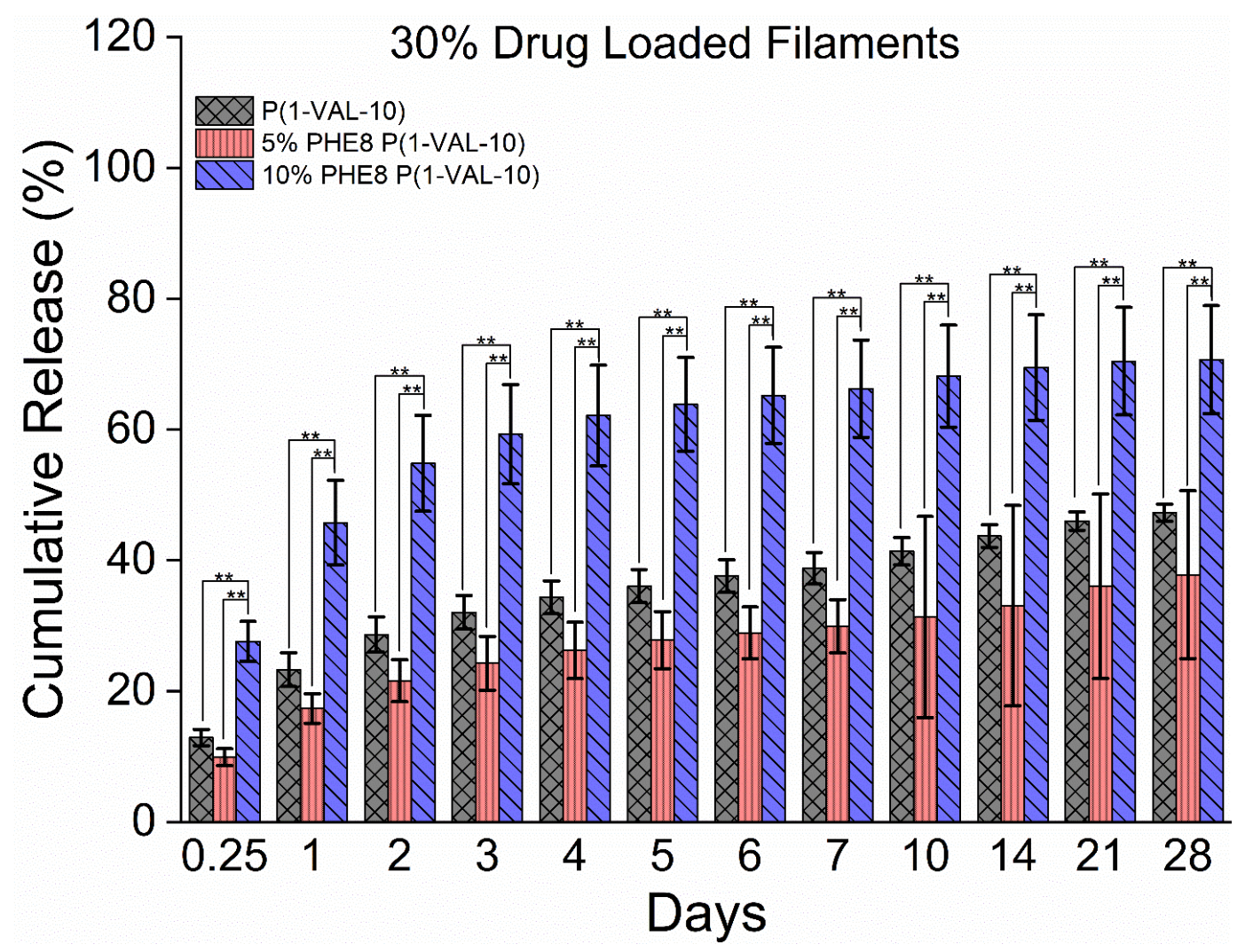

SI Figure 15. Statistical analysis ( $* * \leq 0.01$ significance value) of release values $(\mathrm{n}=5)$ of the $30 \%$ DL filaments at each timepoint was performed using a two-sample t-test using a Welch correction due to unequal variance between samples. In this instance, release from the 10\% PHE8 P(1-VAL$10)$ is significantly faster than both the P(1-VAL-10) and the 5\% PHE8 P(1-VAL-10). 


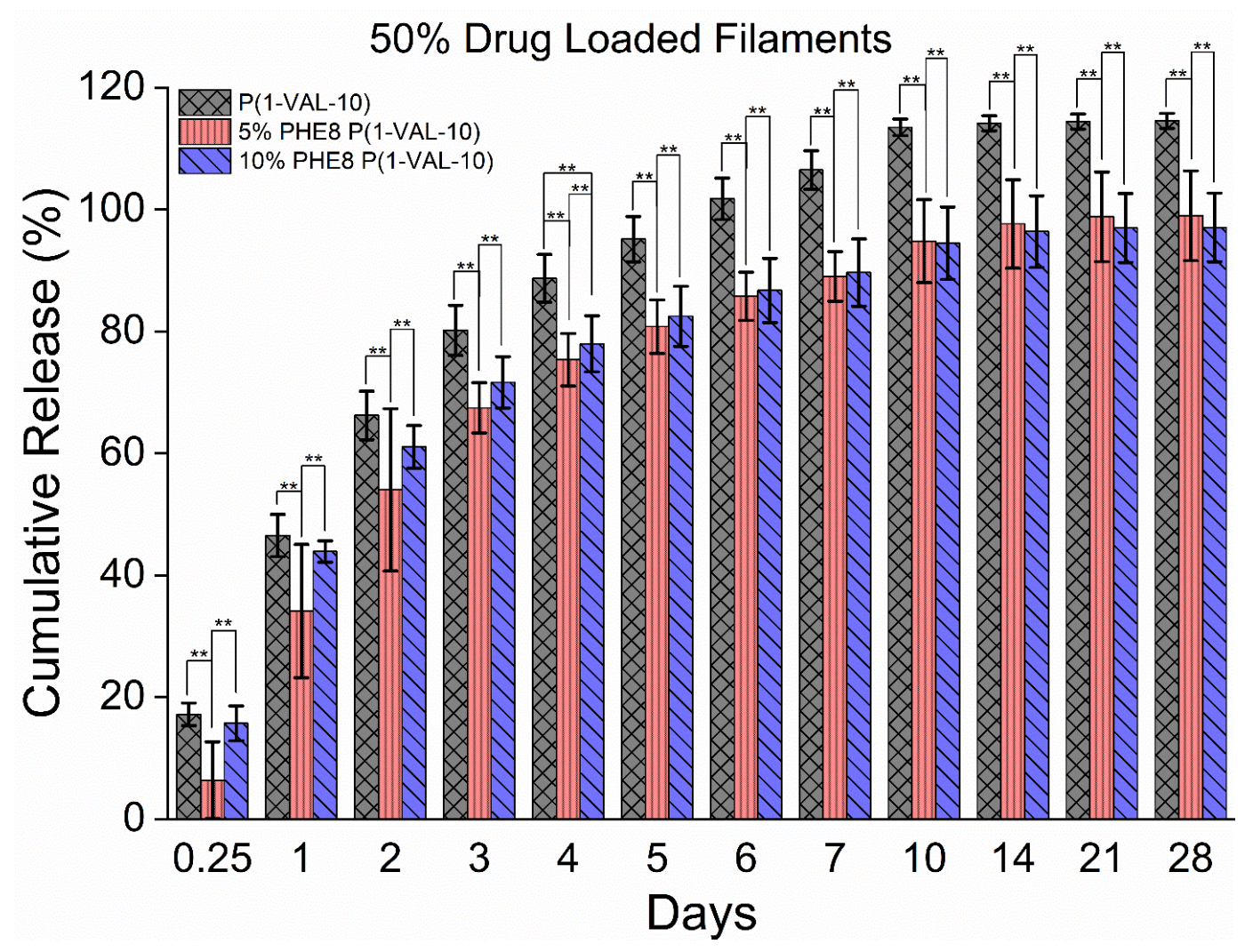

SI Figure 16. Statistical analysis $(* * \leq 0.01$ significance value) of release values $(\mathrm{n}=5)$ of the $50 \%$ DL filaments at each timepoint using a two-sample t-test assuming equal variance shows a significant difference in cumulative release over the 4 week trial period between the samples made with P(1-VAL-10) and the copolymers containing 5\% PHE8 and 10\% PHE8. 

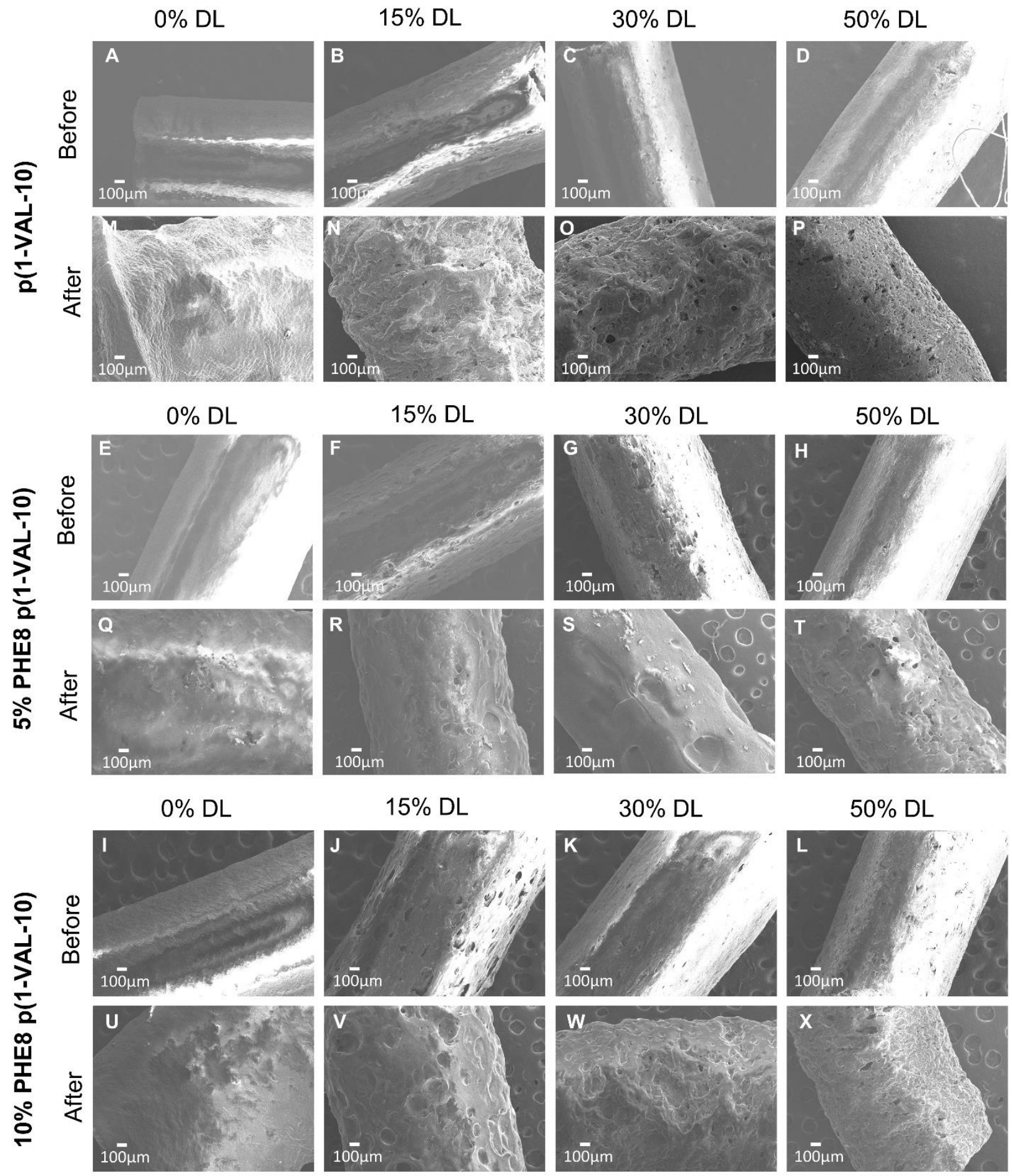

SI Figure 17. SEM images of polymer filaments with varied drug content both before (A-L) and after (M-X) the 4-week release period. Before release, the $0 \%$ and 50\% DL filaments exhibit the smoothest surfaces. After release, all of the filaments display an increase in surface roughness, pitting, and undulations. 
Release Timepoints for 5\% PHE8 P(1-VAL-10) Filaments

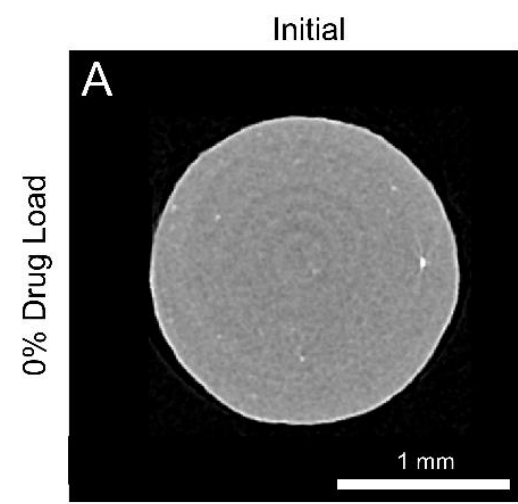

Two Weeks
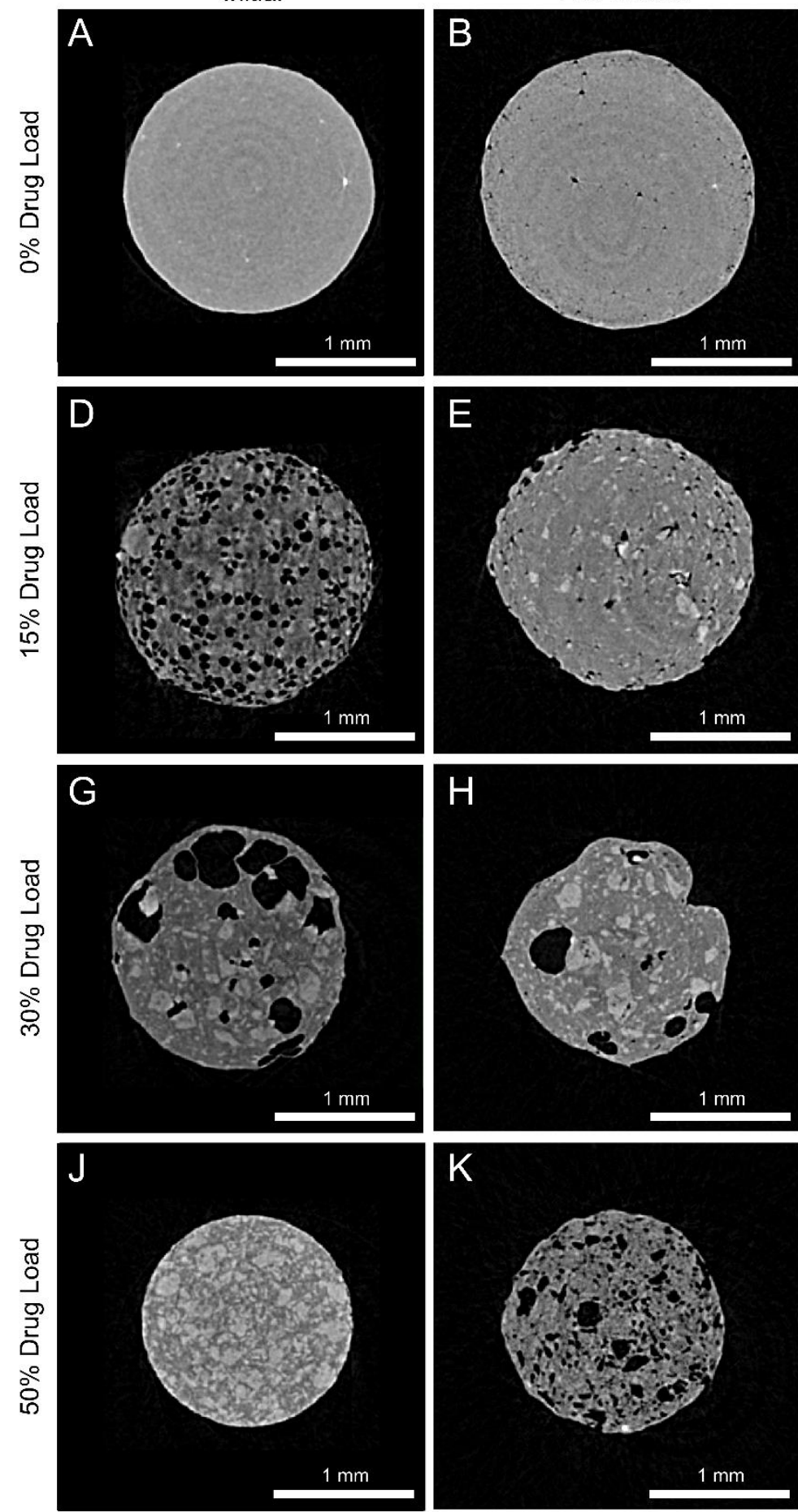
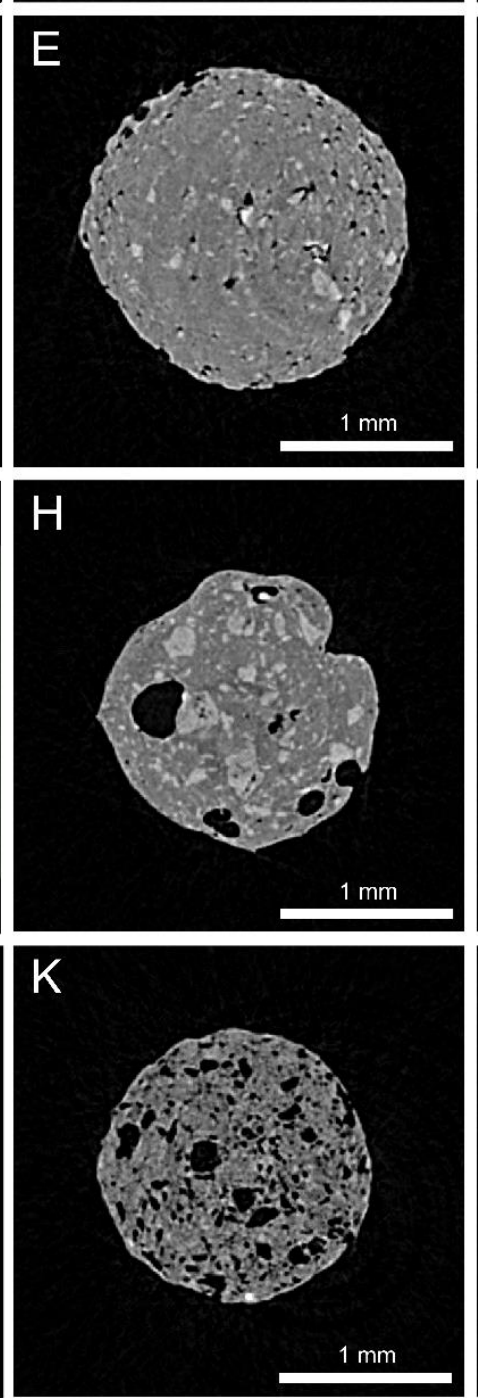

Four Weeks
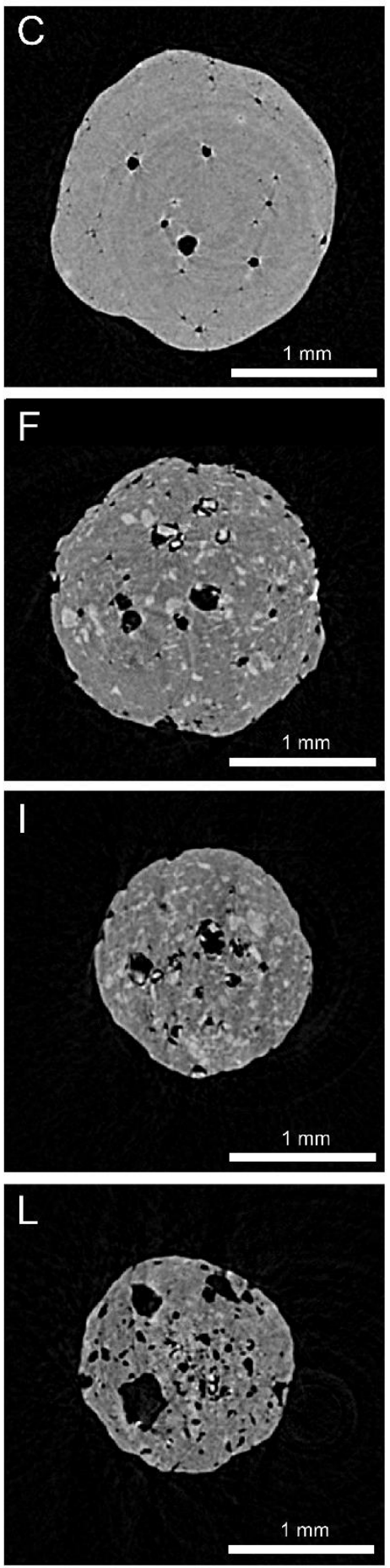

SI Figure 18. Micro-CT cross sections of filaments make from 5\% PHE8 P(1-VAL-10) with entecavir drug loading ranging from 0-50 wt.\%. 
Release Timepoints for 10\% PHE8 P(1-VAL-10) Filaments
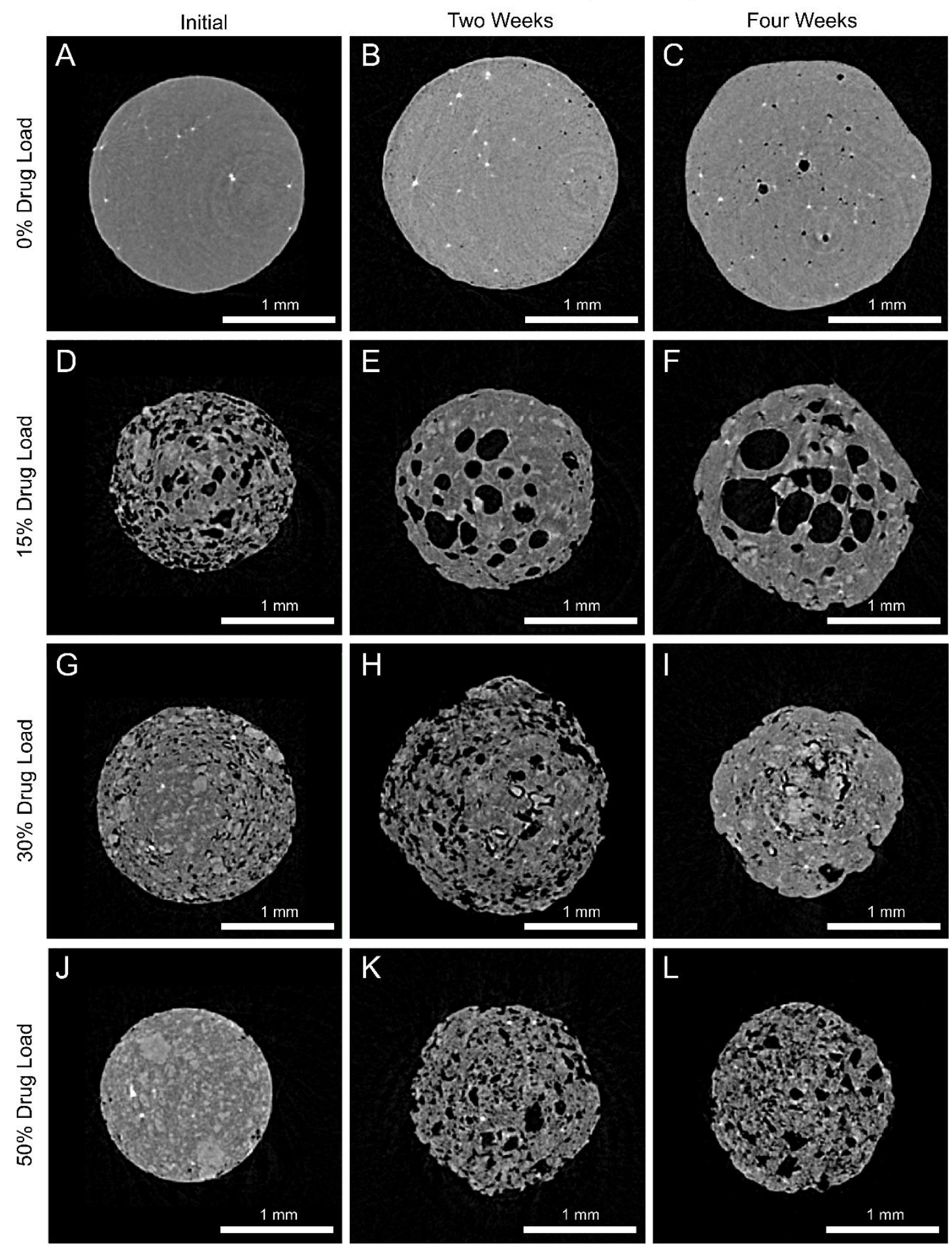

SI Figure 19. Micro-CT cross sections of filaments make from 10\% PHE8 P(1-VAL-10) with entecavir drug loading ranging from 0-50 wt.\%. 


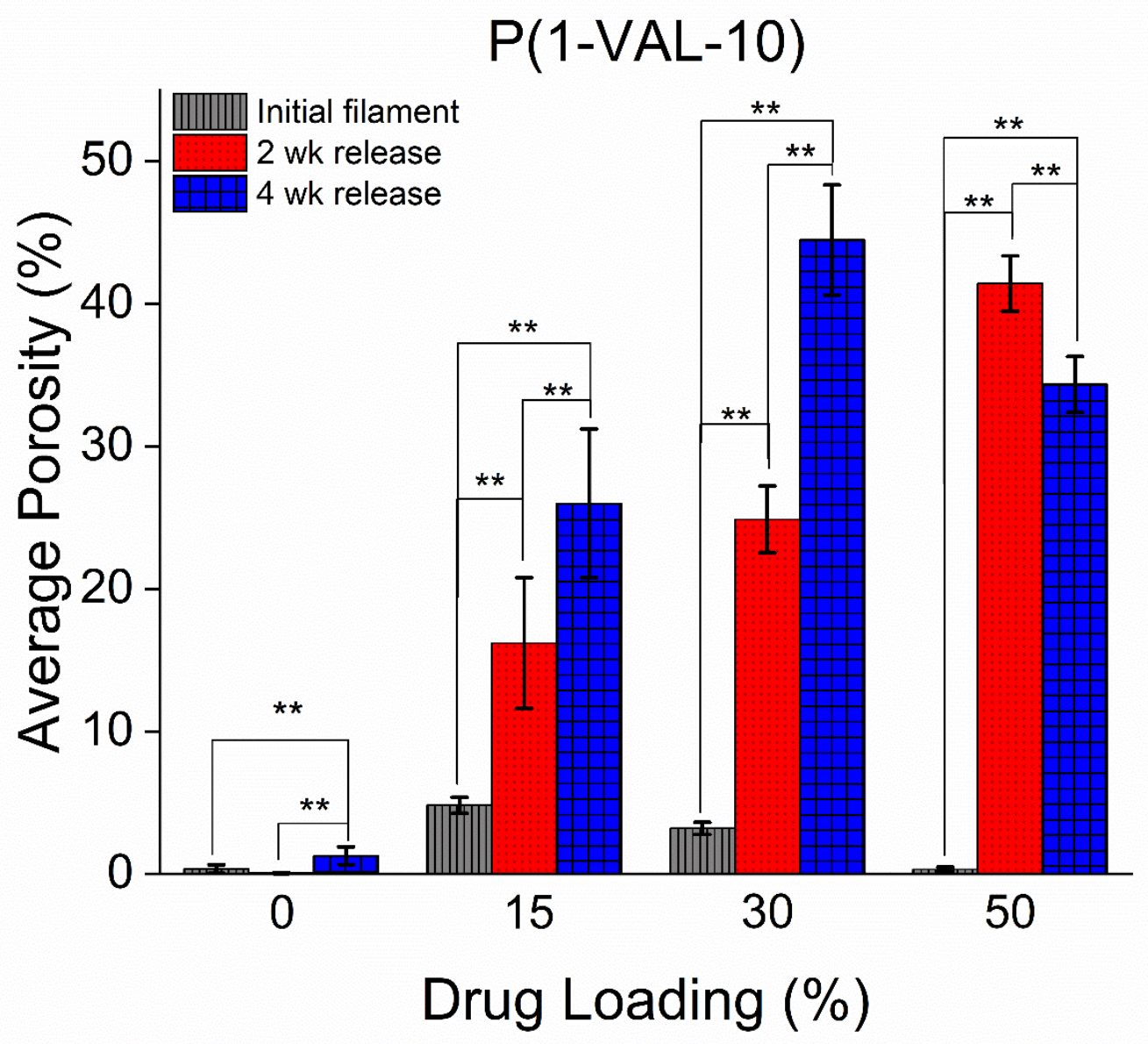

SI Figure 20. Statistical analyisis ( $* * \leq 0.01$ significance value) of sample porosity was performed via a two-sample t-test using a Welch correction due to unequal variance between samples. Filament porosities were calculated from $\mu$-CT cross sections $(n=10)$ of samples before, 2 weeks and 4 weeks into the release period. The porosities increased significantly at 2 weeks and 4 weeks when compared to the initial samples. This confirms that API is being released from the filaments. 


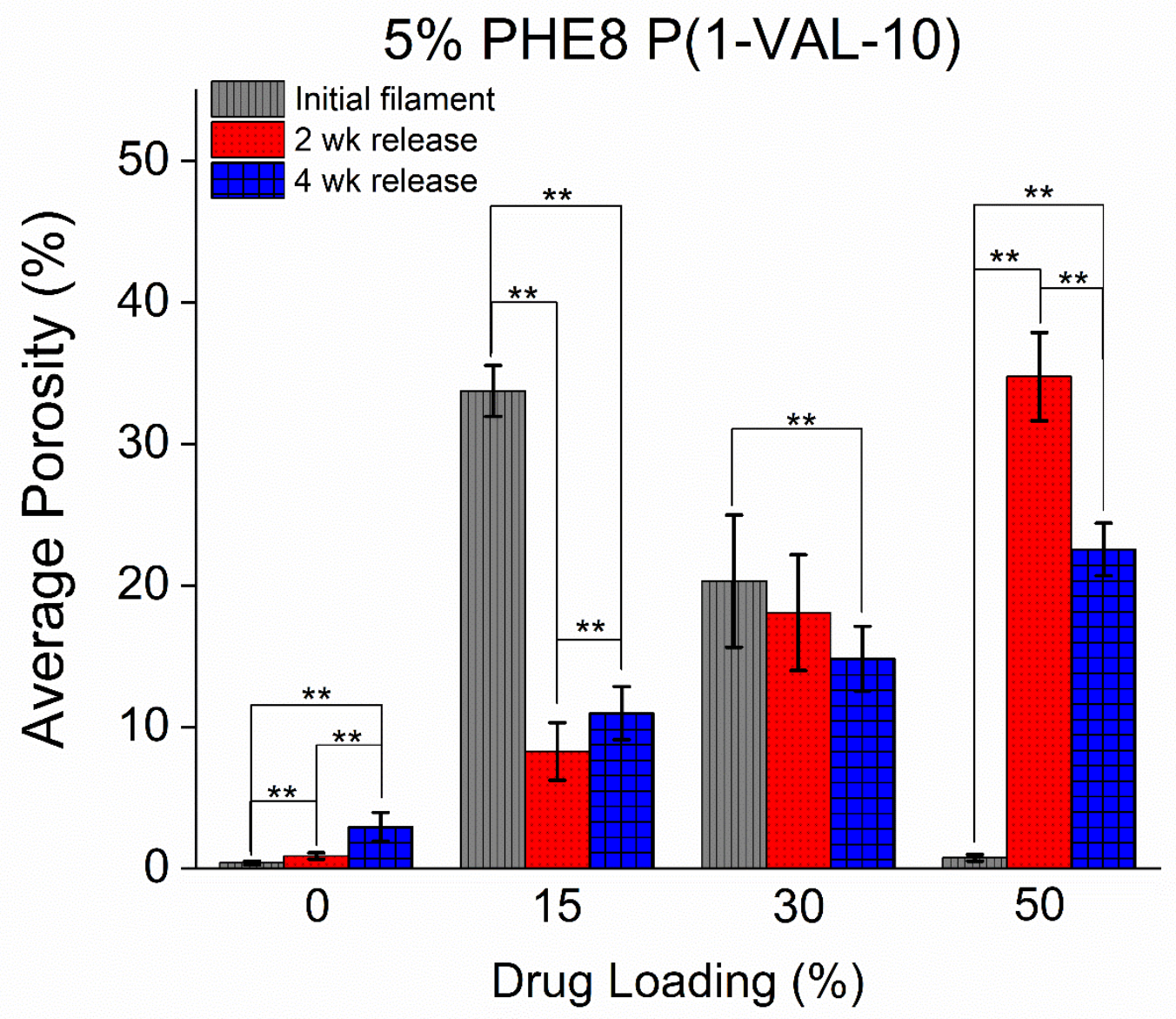

SI Figure 21. Statistical analyisis ( $* * \leq 0.01$ significance value) of sample porosity was performed via a two-sample t-test using a Welch correction due to unequal variance between samples. Filament porosities were calculated from $\mu$-CT cross sections $(n=10)$ of samples before, 2 weeks and 4 weeks into the release period. The porosities of the $0 \%$ DL and 50\% DL filaments increased significantly at 2 weeks and 4 weeks when compared to the initial samples. The porosities of the $15 \%$ and $30 \%$ DL filaments were higher initially when compared to the other timepoints, possibly due to the inability of gases to migrate through the polymer matrix. 


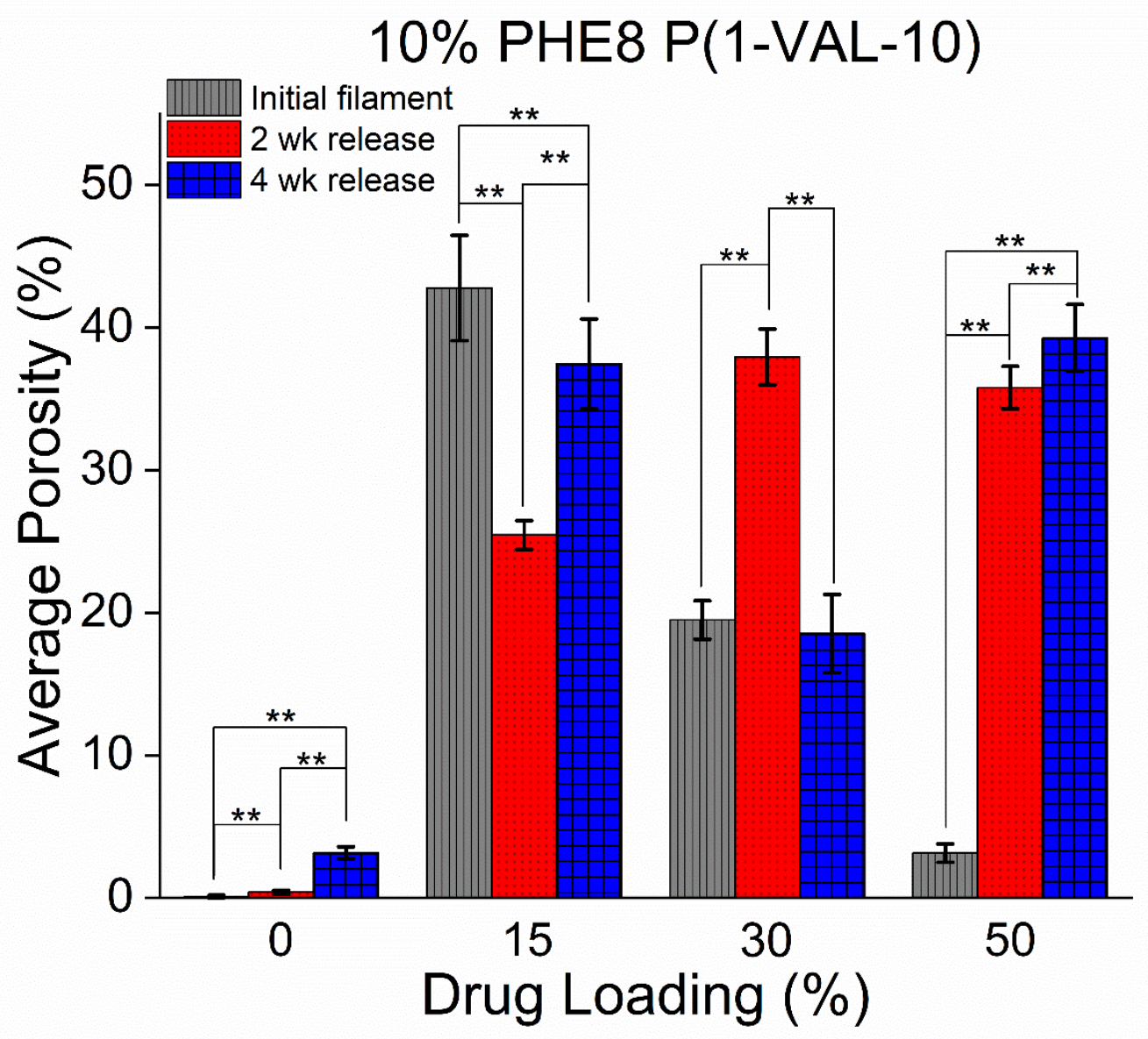

SI Figure 22. Statistical analyisis (** $\leq 0.01$ significance value) of sample porosity of filaments made with $10 \%$ PHE8 P(1-VAL-10) was performed via a two-sample t-test using a Welch correction due to unequal variance between samples. Filament porosities were calculated from $\mu$ CT cross sections $(n=10)$ of samples before, 2 weeks and 4 weeks into the release period. The porosities of the $0 \%$ DL and 50\% DL filaments increased significantly at 2 weeks and 4 weeks when compared to the initial samples. The porosity of the $15 \%$ filament was higher initially when compared to the other timepoints, possibly due to the inability of gases to migrate through the polymer matrix. Larger porosities at the 2 weeks timepoint in the $30 \%$ DL filament could be attributed to filament swelling. 

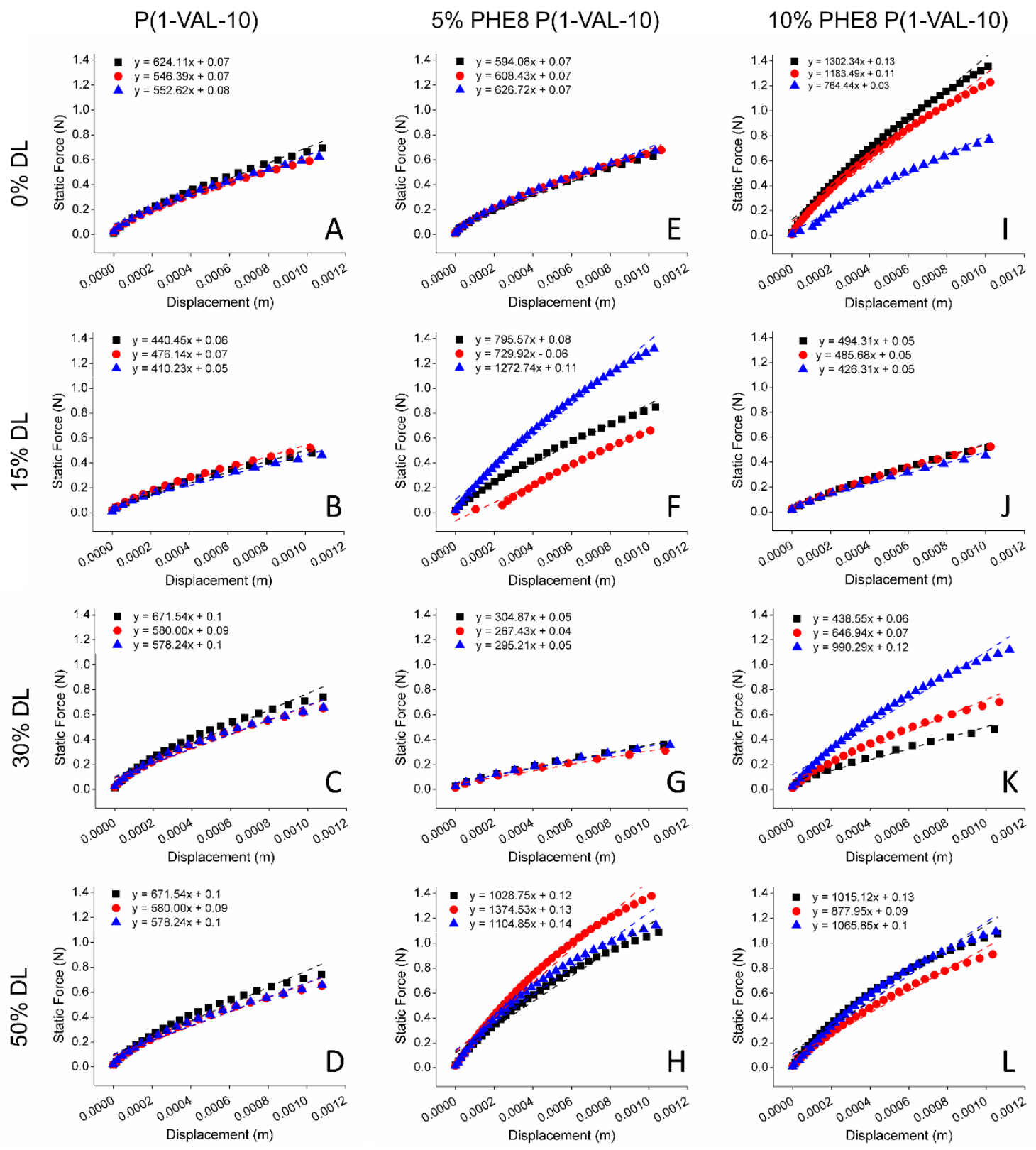

SI Figure 23. Static force vs. displacement profiles for filaments made from P(1-VAL-10) (AD), 5\% PHE8 P(1-VAL-10) (E-H), and 10\% PHE8 P(1-VAL-10) (I-L) at DL varying from 0-50 wt.\%. 

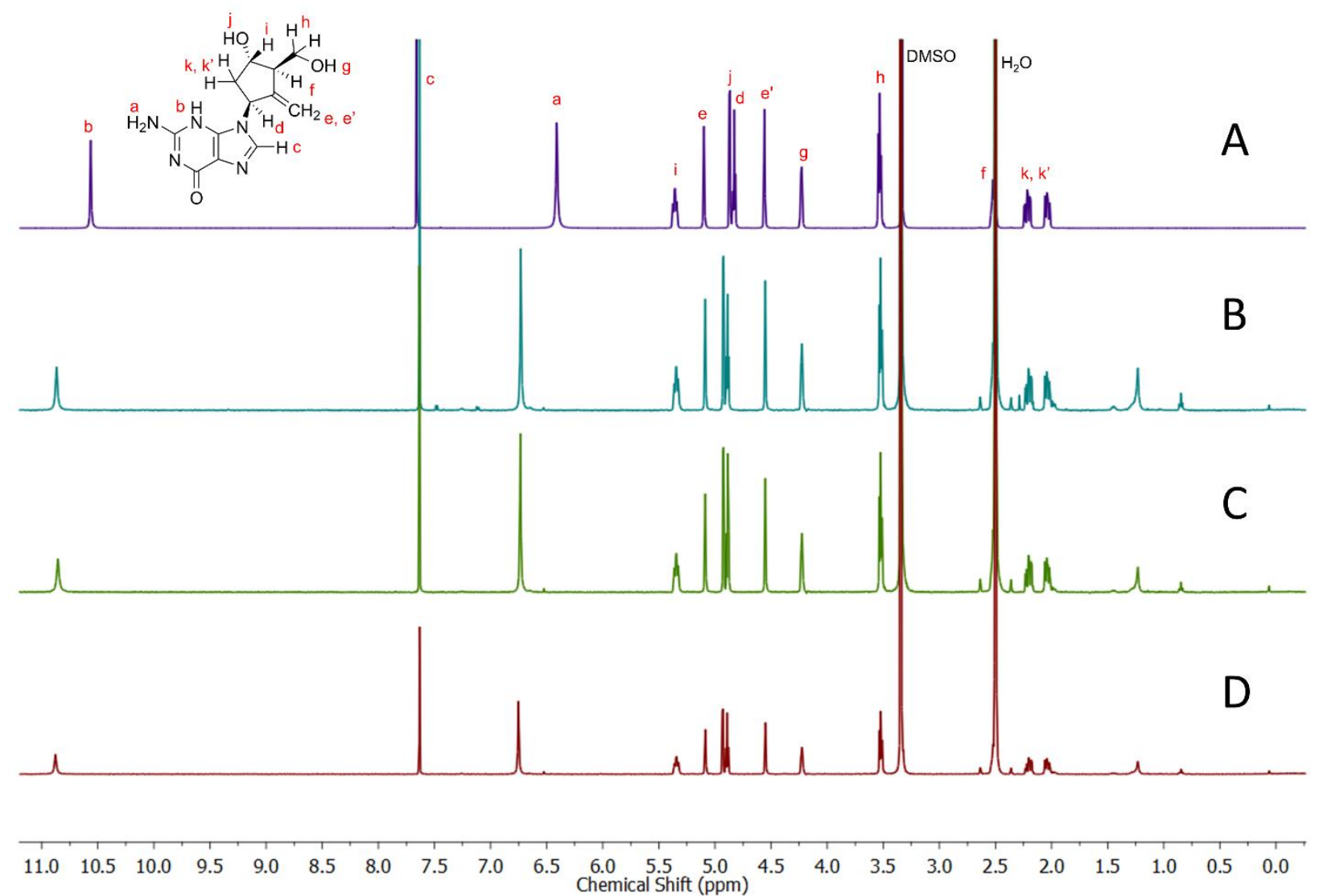

SI Figure 24. ${ }^{1} \mathrm{H}$ NMR of A) pure entecavir monohydrate and freeze-dried effluent from five days of release of 50\% DL B) P(1-VAL-10), C) 5\% PHE8 P(1-VAL-10), and D) 10\% PHE8 P(1VAL-10). Small peaks between 0.5-1.25 ppm are attributed to buffer residue. 